\title{
The importance and value of reporting guidance for scoping reviews: A rehabilitation science example
}

\section{AUTHORS}

ERIN MILLER MScPT, MHM, PhD Candidate, $\mathrm{PT}^{1}$ HEATHER COLQUHOUN PhD, OT ${ }^{2}$

\section{CORRESPONDING AUTHOR}

ERIN MILLER Rehabilitation Sciences Institute, 160-500 University Avenue, Toronto, Ontario, Canada, M5G 1 V7. Email: erin.miller@utoronto.ca
1 Rehabilitation Sciences Institute, University of Toronto, Ontario, Canada.

2 Occupational Science and Occupational Therapy Department, University of Toronto, Ontario, Canada.

\section{ABSTRACT}

Objective: Scoping reviews use a systematic approach to synthesize a body of knowledge. The use of scoping review methodology is increasingly common. Despite recommendations to guide the conduct of scoping reviews, inconsistencies exist with regards to their methodology and reporting. In this case-study, we reflect on our experience using the PRISMA Extension for Scoping Reviews (PRISMA$\mathrm{ScR}$ ): Checklist and Explanation to improve reporting for a scoping review we initially conducted prior to the release of the PRISMA-ScR reporting guidelines.

Study Design and Methods: We evaluated the scoping review against 22 criteria included in the PRISMA-ScR. For each criterion, we provided a rating representing the degree to which we felt the scoping review met the individual criterion in the PRISMA$\mathrm{ScR}$. We also provided comments to substantiate our ratings, along with recommended revisions for the scoping review and considerations for future scoping reviews.

Results: We identified a number of strengths in the initial reporting of our scoping review, as well as opportunities for improvement. The most substantial areas for improvement included the protocol registration, data items and data charting process.
Based on our evaluation, we made revisions to the scoping review manuscript to improve our reporting.

Conclusion: Our evaluation helps to highlight the value of using reporting guidelines to improve reporting of scoping reviews, while also exposing several challenges. In future, we recommend consulting the guidelines during the initial preparation of the scoping review manuscript rather than retrospectively.

What is already known about the topic?

- The use of scoping review methodology is becoming increasingly common.

-While recommendations to guide the conduct of scoping reviews have been published, inconsistencies exist in both scoping review methodology and reporting.

What this paper adds:

- Despite some challenges, we found the PRISMAScR to be an effective tool to guide a structured reflection on our scoping review reporting.

- We encourage authors completing scoping reviews to make use of the PRISMA-ScR to guide their own scoping review reporting.

Keywords: Rehabilitation Research; Interdisciplinary Research; Research Design 


\section{OBJECTIVE}

In this case study we reflect on our experience using scoping review guidelines - at the time recently published - to improve reporting for a completed but unpublished scoping review we had conducted. Specifically, we outline the process used to objectively evaluate our scoping review reporting using the PRISMA Extension for Scoping Reviews (PRISMAScR): Checklist and Explanation, share improvements that resulted from this process, and comment on our PRISMA-ScR user experience. In doing so, we highlight the value of using these reporting guidelines to improve reporting consistency for scoping reviews, while also exposing several potential challenges.

\section{BACKGROUND}

Scoping reviews use a systematic approach to synthesise a body of knowledge. Scoping review methodology lends itself to exploring the extent and nature of research in a given area, establishing the need for a systematic review, providing a means of summarizing and disseminating a range of research findings, and identifying gaps in knowledge. ${ }^{1}$ Within the past decade, there has been a rapid increase in the number of scoping reviews being conducted. ${ }^{2}$

Our scoping review, focusing on competence assessment for airway suctioning, was initially guided by Arksey and O'Malley's seminal framework for the conduct of scoping reviews, and incorporated additional recommendations proposed by Levac et al. ${ }^{1,3}$ Airway suctioning is one technique used by nurses and other health care professionals to remove retained pulmonary secretions. While it can be a necessary element of patient care, caution must be used when performing this technique to avoid causing harm. ${ }^{4}$ In our scoping review, we examined the nature and extent of research on the assessment of clinical competence for health care professionals who perform airway suctioning in adults. ${ }^{5}$ We specifically selected a scoping review methodology given the broad nature of the review, the lack of previous reviews in this area, and the expected methodological variability across relevant studies. ${ }^{6}$

Despite published recommendations to guide the conduct of scoping reviews, ${ }^{1,3,6}$ inconsistencies have been reported with regards to both scoping review methodology and reporting., ${ }^{2,7}$ The 2009 PRISMA statement provides a detailed checklist of items to include for the reporting of systematic reviews and meta-analyses. ${ }^{8}$ When we first conducted our scoping review in early 2018, similar guidelines for scoping reviews were not yet available. However, shortly after preparing a copy of our scoping review manuscript, but prior to its submission to a journal, the PRISMA-ScR was published. ${ }^{9}$ Included in the 27-item PRISMA-ScR are 20 essential items for reporting on scoping reviews and two optional items; the remaining five items are from the original PRISMA checklist and are not applicable to scoping reviews. In the interest of striving to improve reporting in our own scoping review, we took the opportunity to use the newly published reporting guidelines as a tool to guide a structured reflection on our work, and have documented this process.

\section{METHOD}

An objective evaluation of our scoping review was conducted against the 22 relevant criteria included in the PRISMA-ScR. For the evaluation, we used a version of the manuscript we had prepared for submission to a peerreviewed journal. For each item, we provided a rating representing the degree to which we felt our scoping review met that individual criterion. To do this, the lead scoping review author (EM) considered each of the criteria in the reporting guidance against the reporting in our review. The assigned score for each item ranged from 1 to 5 , with 1 representing poor alignment with an individual criterion and 5 representing excellent alignment. In addition, author EM provided comments to substantiate each rating, along with recommended revisions to improve the consistency of our reporting. The draft ratings, comments and recommendations were presented to an interdisciplinary group of health care professionals as part of the oral defense for a doctoral level comprehensive exam. This interdisciplinary group included two physical therapists, one nurse and one occupational therapist, all of who hold university level faculty appointments and had experience in the conduct of scoping reviews. Two members of the group had been involved in conducting the original scoping review, while two had not. All group members had the opportunity to review the proposed ratings, comments and recommendations in detail, pose questions, provide feedback and voice concerns or disagreements. After making minor modifications to the recommendations, the group achieved general agreement. Based on our findings from this evaluation, we revised our scoping review manuscript prior to submitting it for publication in a peer-reviewed journal.

\section{RESULTS OF OUR EVALUATION}

We identified ten items with opportunity for improvement (i.e. that were assigned a rating less than $5 / 5$ ). Of these items, five were assigned a rating of $4 / 5$, two were assigned a rating of 3/5, two were assigned a rating of $2 / 5$ and one was assigned a rating of $1 / 5$. Eleven out of the 12 remaining items were found to be in excellent alignment with criteria detailed in the PRISMA-ScR and were assigned a rating of 5/5. The exception was item \#19: Critical appraisal of sources of evidence (results); this is an optional item that was not applicable to our scoping review. Based on our evaluation, we identified nine recommended revisions to our scoping review, as well as several considerations for future scoping reviews. Our evaluation is summarized in Table 1. 
TABLE 1: ALIGNMENT OF OUR SCOPING REVIEW WITH ITEMS FROM THE PRISMA-SCR

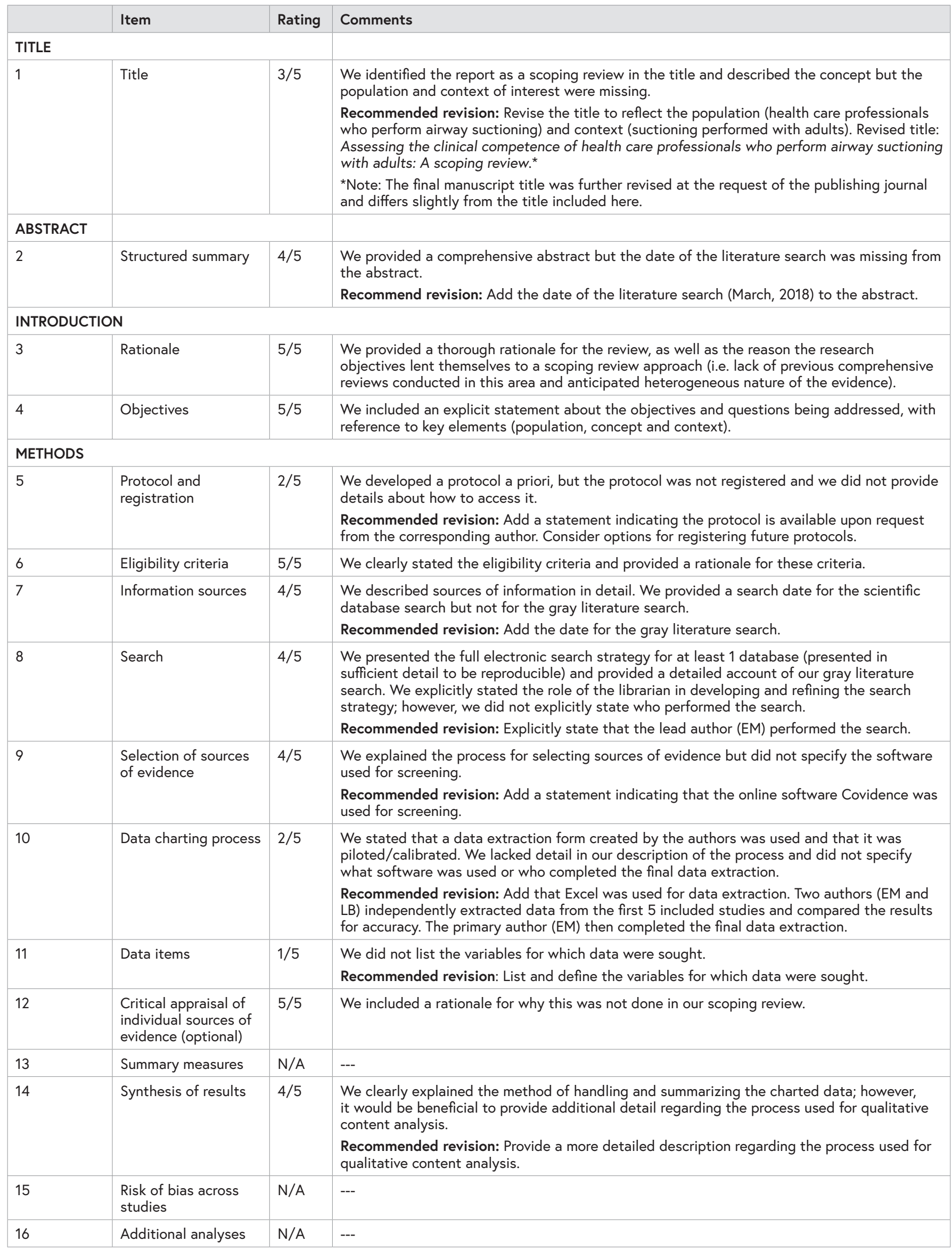


TABLE 1: ALIGNMENT OF OUR SCOPING REVIEW WITH ITEMS FROM THE PRISMA-SCR (continued)

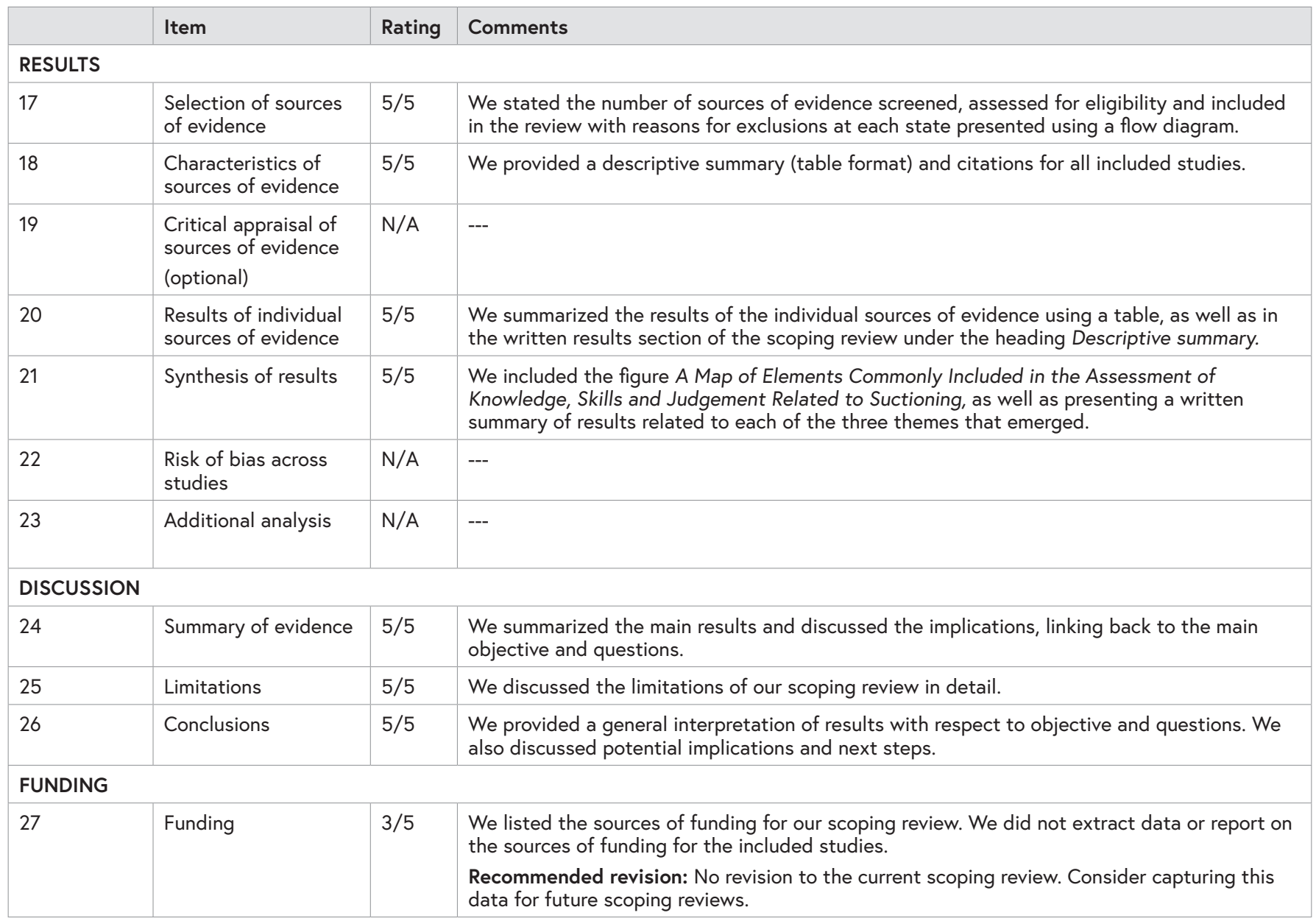

\section{DISCUSSION}

Using a retrospective reflective approach guided by the PRISMA-ScR, we identified a number of strengths in our scoping review reporting, as well as multiple opportunities for improvement. In our discussion, we elaborate on these opportunities for improvement and reflect on our PRISMAScR user experience.

\section{OPPORTUNITIES FOR IMPROVEMENT}

We initially titled our scoping review manuscript Assessing competence for airway suctioning: A scoping review. This title identified our manuscript as a scoping review and included the concept of interest (i.e. the assessment of competence for airway suctioning), but the population (i.e. health care professionals who perform airway suctioning) and context of interest (i.e. airway suctioning performed in adult populations) were not clearly articulated. The concept, population and context of interest serve as the basis for developing the inclusion and exclusion criteria in a scoping review and should be stated concisely in the title. ${ }^{6}$ Following our guided reflection, we revised the scoping review manuscript title to include these important descriptive elements. The final manuscript title was then further revised at the request of the publishing journal. The published title Assessing the clinical competence of health care professionals who perform airway suctioning in adults does not identify the article as a scoping review. This negatively impacts the quality of our scoping review reporting and in retrospect was a missed opportunity for us to engage in further dialogue with the publishing journal advocating for the consistent application of scoping review reporting guidelines.

Our evaluation also identified several other essential details that were missing from the scoping review. For example, while we provided a comprehensive abstract for our scoping review, we failed to include the date of the literature search within the abstract. We also neglected to include the date of our gray literature search in the body of the manuscript. These methodological details were subsequently added in order to facilitate the reader's ability to assess the extent to which the scoping review is up-to-date. ${ }^{9}$

One of the most substantial areas for improvement that we identified concerned our scoping review protocol and registration. Publishing a systematic review protocol can improve methodological transparency and reduce the unintended duplication of a review. ${ }^{10}$ In planning our scoping review, we developed a detailed protocol a priori. 
We initially intended to register this protocol, but struggled to find an appropriate platform to do so. In the end, we proceeded having neither published nor made the protocol publicly available. In our manuscript we made reference to our protocol but did not provide direction on how to access it. To better align with the PRISMA-ScR, we revised our manuscript to include an explicit statement that the protocol would be provided upon request from the corresponding author. This strategy was able to partially mitigate the issues we identified concerning our protocol. However, in the interest of promoting greater transparency and rigor in the conduct of scoping reviews, in future we would endorse a more proactive approach to register or disseminate the protocol in advance of conducting a scoping review. One possibility for this, provided as an example within the PRISMA-ScR, would be to register the protocol with Open Science Framework, a free publicly accessible on-line platform. ${ }^{11}$

Another key area where we identified our scoping review reporting as lacking was with regards to the data items and the data charting process. Levac et al. recommend that two authors independently perform data extraction for the first five to ten records, then convene to ensure their approach is consistent with the aims of the scoping review.3 Similarly, Peters et al. propose the potential need to trial the data extraction form across several studies. ${ }^{6}$ In our own review, two authors independently extracted data from the first five included records, then met to discuss the findings and make minor modifications to the data extraction form. However, we did not list and define all of the variables for which we sought data and failed to describe the full data charting process. Upon reflection, it was clear that neglecting to include this information obscured important methodological details in our review and would impede the reproducibility of our results. Following our guided reflection, we subsequently included these details within our scoping review.

\section{PRISMA-SCR USER EXPERIENCE}

In our situation, the majority items included in the PRISMAScR were found to be clear, concise and easy to apply. One area where we felt that further explanation would have been helpful was with the 'funding' item. Under that item, only minimal explanation and elaboration is provided to support the recommendation to describe funding for the included sources of evidence. The Joanna Briggs Institute (JBI) reviewer's manual takes a less definitive approach, stating that sources of funding for the included studies may be included. ${ }^{12}$ We acknowledge that capturing this data and reporting it could be valuable; for example, if the funding sources for the included studies would conceivably bias their results. For our own scoping review, we did not extract this data or report it as we did not feel that it would impact the interpretation of our findings or add substantial value to our results.
Despite the existence of a wide variety of reporting guidelines [e.g. the CONsolidated Standards of Reporting Trials (CONSORT), ${ }^{13}$ the Strengthening the Reporting of Observational Studies in Epidemiology (STROBE) Statement, ${ }^{14}$ etc.], adherence to reporting guidelines remains suboptimal. ${ }^{15,16}$ Widespread adoption of the PRISMA-ScR guidelines by authors should help improve reporting in scoping reviews, but does not fully address the issue. Adoption of the PRISMA-ScR guidelines by research funders, journals, journal editors and peer-reviewers is also important. ${ }^{17}$ Without this, scoping review authors may continue to experience tensions between reporting guidelines and feedback received during the publication process (e.g. as we did with regards to the title of our scoping review). Finally, because the PRISMA-ScR had not yet been published when we initially conducted our scoping review, we made use of it only after a copy of our manuscript had been completed. A more efficient and practical approach would be to consult the guidelines during initial preparation of the scoping review manuscript. ${ }^{18}$

Given that we evaluated our own scoping review, we acknowledge the lack of author independence. Despite this potential limitation, our evaluation and user commentary help to highlight the value of using reporting guidance to improve the reporting of scoping reviews.

\section{CONCLUSION}

Overall, we found the PRISMA-ScR to be an effective tool to guide a structured reflection on our scoping review reporting. Through this process, we uncovered both areas of strength and opportunities for improvement within our scoping review, strategies to consider for future scoping reviews, and several potential challenges. We would encourage other authors completing scoping reviews to make use of the PRISMA-ScR reporting guidelines.

\section{Implications for research, policy and practice}

Improving consistency and transparency in the reporting of scoping reviews is an important step in promoting scientific rigor across this growing methodology. In discussing our experience using the PRISMA-ScR, we hope to encourage others completing scoping reviews to make use of these reporting guidelines.

Acknowledgements: We would like to thank Dina Brooks, Brenda Mori, Lindsay Beavers and Tracey Collela for their contributions to this project.

Funding Support: This research was supported by the Canadian Lung Association Breathing as One Allied Health Fellowship; the Ontario Respiratory Care Society Fellowship; and the Cardiorespiratory Division - Canadian Physiotherapy Association, administered by the Physiotherapy Foundation of Canada. 
Declaration of conflicting interests: The authors report no conflict of interest; however, we disclose that author HC contributed to the development of the PRISMA-ScR: Checklist and Explanation and is a listed author on that body of work.

\section{REFERENCES}

1 Arksey H, O'Malley L. Scoping studies: towards a methodological framework. Int J Soc Res Methodol. 2005; 8(1):19-32.

2 Tricco AC, Lillie E, Zarin W et al. A scoping review on the conduct and reporting of scoping reviews. BMC Med Res Methodol. 2016; 16(15)

3 Levac D, Colquhoun H, O'Brien KK. Scoping Studies: advancing the methodology. Implementation Sci. 2010; 5(69).

4 American Association for Respiratory Care. AARC Clinical practice guidelines: endotracheal suctioning of mechanically ventilated patients with artificial airways 2010. Resp Care. 2010; 55(6):758-64.

5 Miller EK, Beavers LG, Mori B, Colquhoun H, Colella TJF, Brooks D. Assessing the clinical competence of health care professionals who perform airway suctioning in adults. Resp Care. 2019; 64(7):844-54.

6 Peters MDJ, Godfrey CM, Khalil H, Mclnerney P, Parker D, Soares CB. Guidance for conducting systematic scoping reviews. Int J Evid Based Healthc. 2015; 13(3):141-6.

7 Pham MT, Rajić A, Greig JD, Sargeant JM, Papadopoulos A, McEwen SA. A scoping review of scoping reviews: advancing the approach and enhancing the consistency. Res Synth Methods. 2014; 5(4):371-85.

8 Moher D, Liberati A, Tetzlaff J, Altman DG, The PRISMA Group. Preferred Reporting Items for Systematic Reviews and Metaanalyses: The PRISMA Statement. Ann Intern Med. 2009; 151(4):264-9.

9 Tricco AC, Lillie E, Zarin W, O'Brien KK, Colquhoun H, Levac D, et al. PRISMA extension for Scoping Reviews (PRISMA-SCR): checklist and explanation. Ann Intern Med. 2018; 169(7):467-73.

10 University of York, Center for Reviews and Dissemination: Prospero. [cited 2019 July 28]. Available from: https://www.crd.york.ac.uk/PROSPERO/\#aboutpage

11 Center for Open Science, Open Science Framework. c2011-2019. [cited 31 July 2019]. Available from: https://osf.io/.

12 Peters MDJ, Godfrey C, Mclnerney P, Munn Z, Tricco AC, Khalil H. Chapter 11: Scoping Reviews. In: Aromataris E, Munn Z (Editors). JBI Manual for Evidence Synthesis, JBI, 2020. [cited 2020 June 17]. Available from: https://wiki.jbi.global/ display/MANUAL/Chapter+11\%3A+Scoping+reviews.

13 Schulz KF, Altman DG, Moher D, for the CONSORT Group. CONSORT 2010 Statement: Updated guidelines for reporting parallel group Randomised Trials. Ann Intern Med. 2010; 152(11):726-32.

14 von Elm E, Altman DG, Egger M, Pocock SJ, Gøtzsche PC, Vandenbroucke JP. The Strengthening the Reporting of Observational Studies in Epidemiology (STROBE) Statement: Guidelines for Reporting Observational Studies. Ann Intern Med. 2007; 147(8):573-77.
15 Jin Y, Sanger N, Shams I, Luo C, Shahid H, Li G, et al. Does the Medical Literature Remain Inadequately Described Despite Having Reporting Guidelines for 21 Years?-A Systematic Review of Reviews: An Update. J Multidiscip Healthc. 2018; 11:495-510. doi: 10.2147/JMDH.S155103.

16 Samaan Z, Mbuagbaw L, Kosa, D, Debono VB, Dillenburg R, Zhang S, et al. A Systematic Scoping Review of Adherence to Reporting Guidelines in Health Care Literature. J Multidiscip Healthc. 2013; 6(6):169-88.

17 McGowan J, Straus S, Moher D, Langlois EV, O'Brien KK, Horsley T, et al. Reporting Scoping Reviews- PRISMA ScR Extension. J Clin Epidemiol. 2020; 123:177-9. doi: 10.1016/j. jclinepi.2020.03.016.

18 Lockwood C, Tricco AC. Preparing Scoping Reviews for publication using methodological guides and reporting standards. Nurs Health Sci. 2020; 22:1-4. 